\title{
Metodologías didácticas activas para la enseñanza de las funciones en Educación Secundaria
}

\author{
Eduardo Gregorio Quevedo Gutiérrez ${ }^{\text {y }}$ Alberto Zapatera Llinares ${ }^{b}$ \\ ${ }^{a}$ Universidad de Las Palmas de Gran Canaria, Las Palmas de Gran Canaria, España. \\ equevedo@,dma.ulpgc.es \\ ${ }^{\mathrm{b}}$ Universidad Cardenal Herrera-CEU, CEU Universities, Elche, España. \\ alberto.zapatera@uchceu.es
}

\section{Resumen}

El concepto de función es un elemento fundamental del currículo de matemáticas de la Educación Secundaria Obligatoria, que está presente en otras muchas asignaturas y se utiliza en situaciones de la vida cotidiana en la que se relacionan dos magnitudes, como en estudios de crecimiento poblacional, estudios económicos o fenómenos naturales. Por otra parte, frente al sistema tradicional de enseñanza/aprendizaje es necesario introducir en las aulas dinámicas participativas y colaborativas. En este artículo se plantea un conjunto de metodologías activas complementarias entre sí, que incluyen el método demostrativo, el aprendizaje cooperativo y la gamificación. Se proponen así alternativas didácticas que en último término aproximen al alumno al concepto de función. El reto consiste, por tanto, en discernir qué estrategias podrían ser adecuadas para introducir en los currículos a fin de lograr un aprendizaje significativo y estimular a los alumnos para que deseen seguir aprendiendo.

La materialización de las actividades planteadas se lleva a cabo utilizando el lenguaje de programación Scratch como medio de construcción de la realidad, siguiendo la línea del proceso mental denominado pensamiento computacional. Utilizar la programación con Scratch como vía para el aprendizaje de las Matemáticas permite hacer tangibles y reales contenidos matemáticos de la Educación Secundaria, experimentando de forma práctica y jugando con los efectos, consecuencias y sus resultados asociados. Se toma como punto de partida el hecho de comprender el sistema de coordenadas como un elemento propio de la pantalla de ordenador en la que se trabaja. Las actividades se plantean para el curso de $3^{\circ}$ de ESO, e incluyen las funciones lineal, afín y cuadrática.

Palabras clave: Scratch, Matemáticas, Función, Secundaria, Método Demostrativo, Aprendizaje Cooperativo, Gamificación. 


\section{Introducción}

Un reciente estudio del año 2016 realizado por la Comisión Europea "Developing Computational Thinking in Compulsory Education, Implications for Policy and Practice" (Bocconi et al., 2016), plantea que en la última década, el pensamiento computacional y sus conceptos relacionados (por ejemplo, codificación, programación, o pensamiento algorítmico) están recibiendo una atención creciente en el campo educativo, que ha dado lugar a una gran cantidad de iniciativas de implementación públicas y privadas. Sin embargo, más allá de este interés generalizado, la integración exitosa del pensamiento computacional en la educación obligatoria aún se enfrenta problemas y desafíos no resueltos.

El pensamiento computacional como tal, se enuncia a principios de esta década. Jeannette Wing, doctora en Ingeniería en Informática por el MIT (Massachusetts Institute of Technology), que es una de sus máximas exponentes, presentó una definición en 2010 centrada en el uso de conceptos informáticos para hacer cosas, desde resolver problemas hasta comprender el comportamiento humano, pasando por el diseño de sistemas (Wing, 2010). El pensamiento computacional se basa fundamentalmente en dos teorías del aprendizaje: el constructivismo de Jean Piaget, psicólogo y pedagogo suizo que defendía dotar de herramientas al estudiante para que pueda resolver problemas (Forman y Pufall, 1988), y el construccionismo de Seymour Papert, matemático, informático y educador estadounidense nacido en Sudáfrica, que proponía la construcción de modelos mentales para comprender el mundo que nos rodea (Papert y Harel, 1991). Ambas teorías de aprendizaje se centran en la construcción de elementos, siguiendo la filosofía denominada maker para resolver problemas.

Scratch es un lenguaje de programación visual desarrollado por un equipo del MIT Media Lab liderado por Mitch Resnick, que se utiliza por parte de estudiantes, profesores y padres para crear de forma sencilla animaciones e interacciones, fomentando el pensamiento computacional y poniendo así en práctica las tesis de Piaget y Papert (Marji, 2014). La principal aportación de Scratch es que está destinada a usuarios de temprana edad, lo que lo hace directamente aplicable como herramienta didáctica destinada a enseñar programación en niveles finales de primaria y en secundaria. Cada vez más, los alumnos, desde edades más tempranas, están interesados en la programación como medio creador de diferentes utilidades, aplicaciones y juegos. Scratch ofrece una perspectiva avanzada en conocimiento, pero sencilla en manejo (Corralero, 2011), por lo que actualmente se está usando en el ámbito educativo para una amplia variedad de aplicaciones (Suárez, Andrés y SarmientoPorras, 2015). 
La enseñanza de las funciones lineal, afín y cuadrática en $3^{\circ}$ de Educación Secundaria Obligatoria (ESO), de acuerdo con el currículo educativo actual en España, constituye un reto para el docente. El objetivo consiste en utilizar funciones para representar situaciones reales, simbolizar relaciones y analizar e interpretar críticamente información de gráficas funcionales que aparecen en medios de comunicación o en otras asignaturas.

El presente artículo introduce una alternativa didáctica a la introducción de las funciones haciendo uso de Scratch. Para ello se plantean un conjunto de metodologías activas complementarias entre sí, incluyendo el método demostrativo, el aprendizaje cooperativo y la gamificación. La finalidad es doble, por una parte presentar de forma didáctica el tema tratado y por otra, introducir a los alumnos en la programación con Scratch.

\section{Metodologías didácticas activas}

La motivación de las actividades que se presentarán en este artículo para exponer la enseñanza de las funciones con Scratch se centra en proponer alternativas didácticas que, en último término, aproximen al alumno al concepto de función. De esta forma, se ha planteado el lenguaje de programación Scratch como medio de construcción de la realidad. El reto consiste en discernir qué estrategias podrían ser adecuadas para introducir en los currículos a fin de lograr un aprendizaje significativo.

Así, frente al sistema tradicional de enseñanza/aprendizaje es necesario introducir en las aulas dinámicas participativas y colaborativas, que permitan la interacción de los estudiantes en actividades donde el educador modifica su rol tradicional por el de orientador y el alumno toma un papel más activo aprendiendo a través de su propio esfuerzo (Quevedo-Gutiérrez, Vega-Moreno, Rodríguez-Cabrera y Quintana-Gil, 2016). Se busca por tanto un conjunto de metodologías activas complementarias entre sí, incluyendo el método demostrativo, el aprendizaje cooperativo y la gamificación

\subsection{Método Demostrativo}

El método demostrativo es un método asociado a la estructuración de los contenidos que realiza el docente, cuyas características se presentan en la Figura 1 (Ferrando, 2018). En este caso el profesorado, en función de la práctica, transmite la información al alumnado mediante la demostración de la tarea. Este método encaja muy bien con la idea del pensamiento computacional, ya que se comparte una idea a partir de la cual los alumnos pueden desarrollar la actividad en base a propuestas propias, incluyendo elementos nuevos. 
Así mismo, Scratch permite visualizar en tiempo real la programación de la tarea realizada y su traducción en la ejecución de la misma.

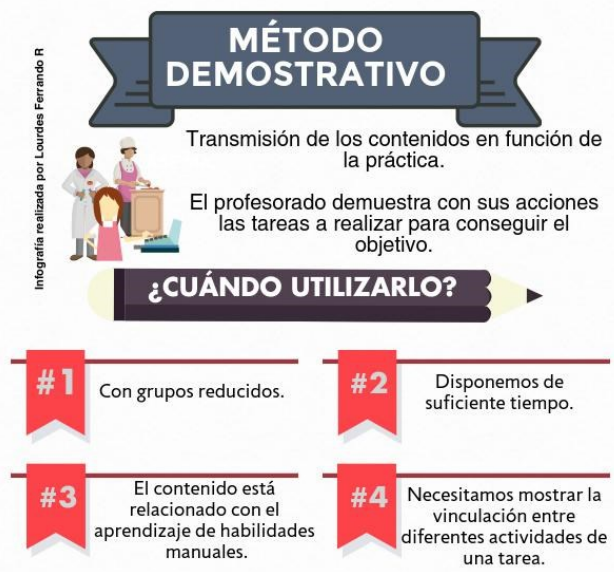

4 PASOS PARA SU PUESTA EN PRÁCTICA



2- Explicación de la tarea

(9) Puntualización de etapas y puntos claves de lo que se va a realizar.

3- Realización de la tarea por parte del profesorado

(-) Realización de la tarea, acompañando con explicaciones verbales. marcando puntos claves $\mathrm{y}^{\text {"trucos }}$ a recordar.

4- Actuación del alumnado.

(i) Cada estudiante realiza la tarea, paso a paso, explicando lo que ve haciendo, siendo cada vez mas autonomo en la ejecución de la misme.

Figura 1.- El Método Demostrativo y 4 pasos para su puesta en práctica (Ferrando, 2018)

\subsection{Aprendizaje Cooperativo}

Tal y como indican Salmerón, Gutiérrez-Braojos, Rodríguez, y Salmerón “el aprendizaje cooperativo se impulsa a mediados del siglo XX como estrategia docente favorecedora de integración escolar..., pero es propuesto y apoyado por los teóricos constructivistas y socioculturales como favorecedor del desarrollo cognitivo y socio-afectivo." (2010, p. 309). El aprendizaje cooperativo consiste en la disposición de pequeños grupos de alumnos que trabajan juntos, a fin de mejorar su aprendizaje. En esta metodología didáctica se identifican tres tipos, según la estabilidad o permanencia del grupo (Johnson y Johnson, 1995):

1. Grupos informales: El profesorado los puede utilizar durante una actividad didáctica específica, de forma que pueden durar desde unos pocos minutos hasta una hora o la duración de una clase. La finalidad es que esta estrategia es la mejora de la atención y la comprensión de la tarea tratada, de modo que el grupo organice, analice, explique e interprete la información de manera adecuada.

2. Grupos formales: Se forman para un periodo amplio de clases, por ejemplo, un trimestre. Su finalidad, como en el caso anterior, es que los estudiantes participen y se ayuden para organizar, analizar e interpretar la información, de manera que cooperen para el logro de los objetivos individuales y colectivos. 
3. Grupos cooperativos de base: Se forman para periodos largos de tiempo, por ejemplo, un curso completo. Se pretende que se establezcan relaciones duraderas de cooperación y ayuda. Así, el grupo sirve de apoyo para que los estudiantes no se rezaguen en su aprendizaje. Por tanto, su objetivo es motivar a los estudiantes, a la par que se les ofrece apoyo permanente a través de sus compañeros.

Tomando en consideración que las actividades planteadas en este artículo para reforzar conceptos relacionados con las funciones quedarían encuadradas en un trimestre determinado, quizás sería adecuado plantear grupos formales en clase, que comiencen a trabajar de forma cooperativa con Scratch. Estos grupos se podrían extender al curso completo, convirtiéndose en grupos cooperativos de base si esta herramienta tecnológica, $u$ otras relacionadas, se utilizan a lo largo del resto del curso y se desea disponer de una visión global del desarrollo de la actividad realizada por los grupos de trabajo. En este caso, sería adecuado monitorizar en todo momento la capacidad de trabajo de los grupos, a fin de introducir cambios cuando fuese necesario. Se considera que un número adecuado para los grupos sería de tres alumnos, pudiendo ser incluso de dos, si los recursos informáticos del centro educativo lo permiten.

\subsection{Gamificación}

Según Zichermann y Cunningham la gamificación consiste en "un proceso relacionado con el pensamiento del jugador y las técnicas de juego para atraer a los usuarios y resolver problemas" (2011, p.11). La combinación del método demostrativo y el aprendizaje cooperativo conjuntamente con la gamificación consigue aplicar de forma colaborativa dinámicas y estrategias del juego al proceso de aprendizaje. Para ello se ha de buscar un mensaje claro y definir la intencionalidad, elegir entonces la estrategia a seguir y finalmente evaluar y medir el progreso. Auqnue el uso del juego ha sido utilizado tradicionalmente en edades tempranas, estigmatizándolo en edades más avanzadas, al considerarse en ocasiones una pérdida de tiempo, en los últimos años se ha convertido en una tendencia metodológica con gran presencia en las aulas (Díez-Rioja, Bañeres-Besora y Serra-Vizern, 2017).

El enfoque llevado a cabo en el aprendizaje de las funciones mediante la programación con Scratch constituye un juego en sí mismo en tanto permite al alumno probar diferentes opciones hasta llegar a la solución del problema, sin miedo a equivocarse en el proceso. Esto es muy interesante ya que el hecho de no ser capaz de llegar a la solución final directamente puede suponer en sí mismo la motivación para que el estudiante quiera seguir jugando y mejorando; por el contrario, en el sistema educativo normalmente las equivocaciones se penalizan, lo cual puede llevar a la desmotivación (Zamora y Ardura, 2014). 


\subsection{Combinación de metodologías planteadas}

La utilización del aprendizaje cooperativo combinado con el método demostrativo y la gamificación proporcionaría las siguientes ventajas:

1. Promueve el desarrollo cognitivo y socio-afectivo de los miembros del grupo, basándose en una estrategia de trabajo que orienta hacia la solidaridad a través del juego como elemento de motivación adicional.

2. Implica el desarrollo de competencias de trabajo en equipo como son la comunicación, la interacción, la cooperación, el compromiso, la responsabilidad o el liderazgo.

3. Reorienta el esfuerzo competitivo individual hacia usos positivos de colaboración para el logro de objetivos individuales y colectivos. La integración del método demostrativo implica además una mayor conexión con el docente.

\section{Actividades planteadas}

Partiendo de la utilización de las metodologías anteriormente comentadas, se presentan dos actividades haciendo uso del lenguaje de programación Scratch; la primera de ellas está referida a las funciones lineal y afín, mientras que la segunda trata la función cuadrática.

\subsection{Actividad 1: Yendo al cine con las funciones lineal y afín}

En este apartado se presenta una actividad aplicada a una función lineal, que se adapta entonces a una función afín, partiendo de una situación de la vida cotidiana en base a un problema de estructura multiplicativa de razón. Este tipo de problemas se identifican con una regla de tres en la que existe proporcionalidad directa y en los que una forma de resolución didáctica adecuada es la reducción a la unidad. Se plantea para ello el siguiente problema:

"Si a 3 amigos ir al cine nos cuesta $18 €$, ¿cuánto nos costará ir al cine a 7 amigos?"

Según la técnica que se suele explicar en clase para la regla de tres directa, se realizaría un producto cruzado, de la siguiente forma:

$$
\begin{gathered}
3 \text { amigos } \rightarrow 18 € \\
7 \text { amigos } \rightarrow \mathrm{x} €
\end{gathered}
$$

Por lo que entonces $3 \cdot \mathrm{x}=7 \cdot 18 \rightarrow \mathrm{x}=42 €$ 
Esta forma de resolución se encuentra alejada de la forma práctica de cálculo que se lleva a cabo en la vida real, que pasaría por preguntarse cuánto cuesta una entrada para entonces multiplicar el coste de una entrada por el número de amigos que van a ir al cine, esto es:

Si a 3 amigos ir al cine le cuesta $18 €$, la entrada cuesta $18 / 3=6 €$

Entonces a 7 amigos ir al cine les costaría $7 \cdot 6=42 €$

Este problema aplicado a las funciones podría entenderse entonces como una función lineal en la que la pendiente constituiría el coste de la entrada (6 €), la variable independiente $x$ sería el número de amigos y la variable dependiente $y$ sería el coste total para ir al cine. Partiendo de esta idea se podría plantear un programa con Scratch de la siguiente forma:

1. Se plantea el ejemplo anterior con 7 amigos, haciendo ver a los estudiantes lo práctica que es la resolución por reducción a la unidad.

2. Se hace pensar a los estudiantes en el marco con el cual se va a trabajar el problema para representar una función (la pantalla de Scratch), y se les hacen las siguientes preguntas: ¿en qué cuadrantes del sistema de coordenadas puede encontrarse la solución del problema? ¿cuál sería el número máximo de amigos que podemos considerar para que el resultado quede dentro de la pantalla de Scratch?

3. Se diseña un programa en Scratch en el que se dibuje la función comentada anteriormente y en el que además se pregunte cuántos amigos van a ir al cine y en base a ello responda y marque la solución del problema en la función.

4. Se modifica el programa para que el usuario pueda introducir el coste de la entrada.

Los estudiantes podrán comprobar de forma sencilla que el resultado del problema se va a encontrar siempre en el primer cuadrante, lo cual ocurre mucho en problemas del vida real, y podrán plantearse entonces el número máximo de amigos que se puede considerar para que la solución quede dentro de la pantalla de Scratch. Para plantear este ejercicio se puede tomar como referencia el escenario $x y$-grid-30px, con el que los estudiantes pueden ver que el valor máximo quedaría establecido para $\mathrm{x}=30$ e $\mathrm{y}=180$, tal y como se representa en la Figura 2 (la cruz marca la posición para $\mathrm{x}=90$ e $\mathrm{y}=90$ y cada cuadrado de la cuadrícula es de 30x30). Por tanto el número máximo de amigos sería de 30. 

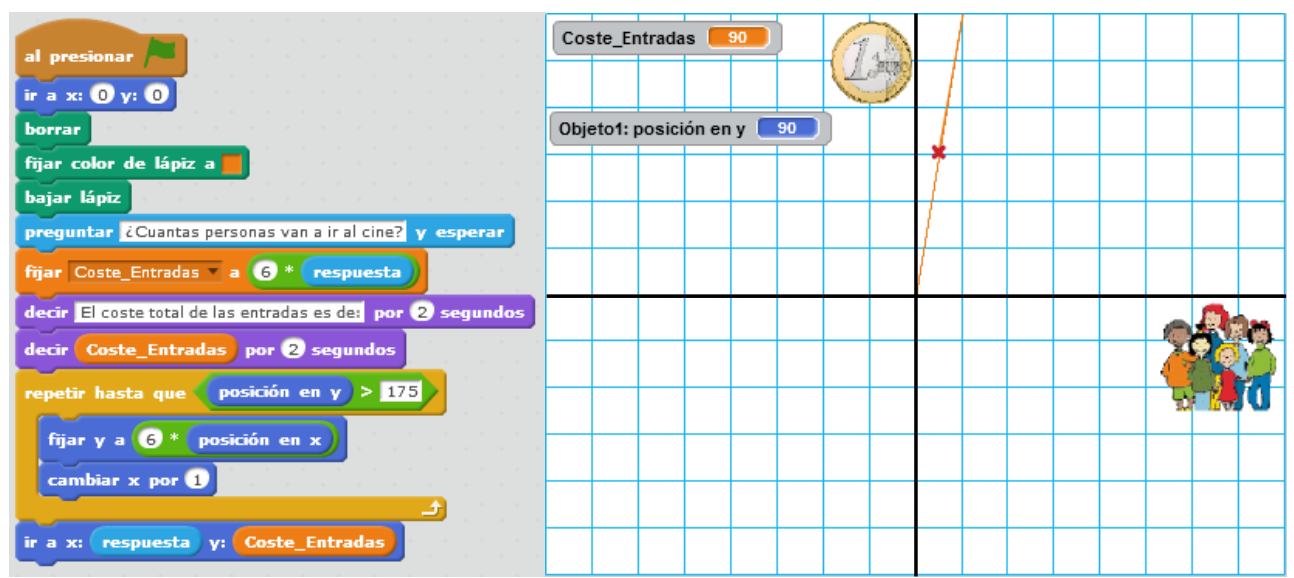

Figura 2.- Función lineal con la solución marcada para 15 amigos (coste $=90 €)$

Se puede modificar entonces el programa de la Figura 2 para que el alumno pueda introducir el coste de la entrada. Únicamente habrá que definir una nueva variable a la que para asignar su valor al principio del programa. Entonces se podría introducir una pequeña variante para pasar de la función lineal $y=m x$ a la función afín $y=m x+n$, dotando de significado a la ordenada en el origen $n$, pensando en las siguientes alternativas:

1. En el día del cine, cuando las entradas están a $2 €$, y dada la alta demanda, para evitar una compra masiva de entradas por Internet, se ha puesto un sobrecoste a la compra (sobre el total) de $15 €$, cuando el número de entradas compradas es igual o mayor a 15.

2. En el día del cine, cuando las entradas están a $2 €$, un cine en crisis ha decidido para fomentar aún más la compra una rebaja a la compra (sobre el total) de $15 €$, cuando el número de entradas compradas es igual o mayor a 15.

Se representan en la Figura 3 las funciones afines correspondientes a las alternativas 1 y 2 , que aparecen respectivamente, por encima y por debajo de la función lineal representada.

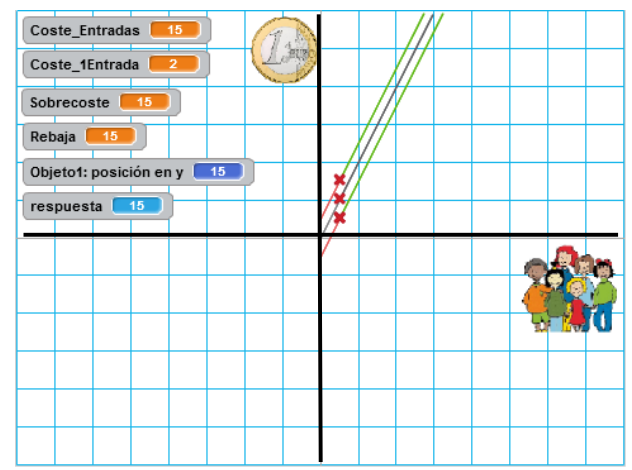

Figura 3.- Representación de funciones afines frente a lineal 


\subsection{Actividad 2: Encestando con la función cuadrática}

Muchas veces los estudiantes al afrontar la resolución de ecuaciones de segundo grado del tipo $y=a x^{2}+b x+c=0$ aplican la fórmula que proporciona las posibles soluciones sin comprender su significado gráficamente, nos referimos a la siguiente expresión:

$$
x=\frac{-b \pm \sqrt{b^{2}-4 a c}}{2 a}
$$

De esta expresión se puede entender que, cuando $b^{2}-4 a c=0$, existirá un punto central en el eje de abscisas (eje $x$ ) a partir del cual habrá un desplazamiento igual tanto a izquierda como a derecha, que determinará los puntos de corte con el eje $x(y=0)$ siempre y cuando $b^{2}>4 a c$ (para que existan soluciones reales). Este punto se denomina vértice y su componente en el eje $x$ es por tanto igual a $x_{v}=-b / 2 a$, por lo que la expresión en el eje $y$ $\left(\mathrm{y}_{\mathrm{v}}\right)$ vendrán por tanto dada por la siguiente expresión:

$$
y_{v}=a x_{v}^{2}+b x_{v}+c=a \cdot\left(\frac{-b}{2 a}\right)^{2}+b \cdot \frac{-b}{2 a}+c=\frac{b^{2}}{4 a}-\frac{b^{2}}{2 a}+c=-\frac{b^{2}}{4 a}+c
$$

En la Figura 4 se muestra un ejemplo de las características comentadas para la función $y=-x^{2}+6 x+16$. En esta actividad se tratará de discernir la posición del vértice en base a su expresión aproximando la solución a partir de un problema básico de tiro parabólico, que aunque los estudiantes no realizarán en Física hasta $1^{\circ}$ de Bachillerato, servirá para ir adquiriendo un conocimiento básico de su funcionamiento.

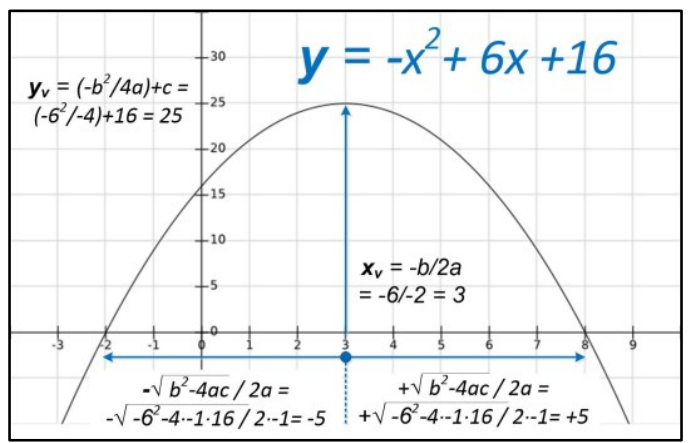

Figura 4.- Función cuadrática con cálculo de vértice y puntos de corte con el eje $X$

Partiendo de un programa sencillo en Scratch y de una escenografía adecuada, se puede simular el lanzamiento de una pelota de baloncesto generando una parábola, tal ycomo se representa e la Figura 5. El reto consistirá en plantear a los estudiantes que obtengan valores adecuados para los coeficientes $a, b$ y $c$ a partir de lo expuesto anteriormente. 

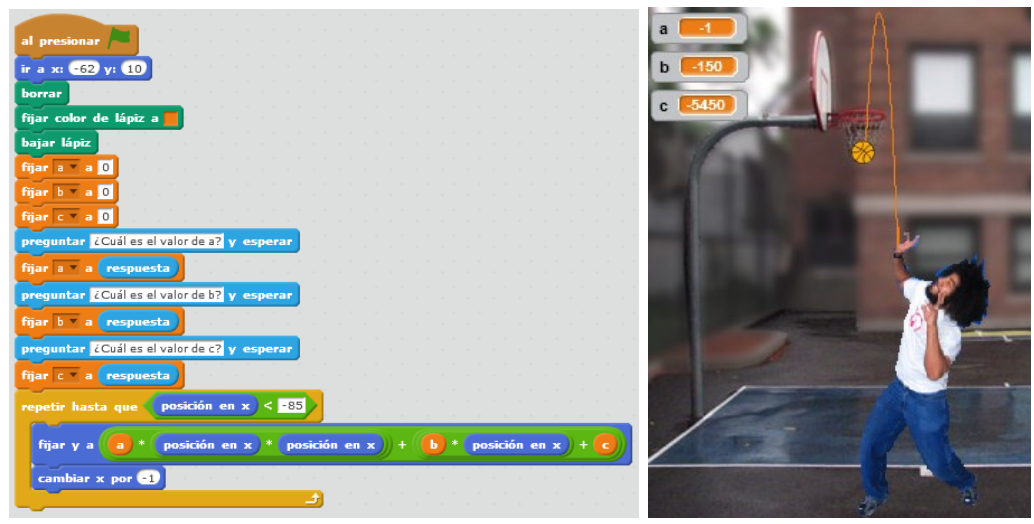

Figura 5.- Programa en Scratch y parábola correspondiente a coeficientes $a=-1, b=-150$ y $c=-5450$

\section{Conclusiones}

En este artículo se han presentado dos actividades innovadoras orientados al bloque de Funciones de la asignatura de Matemáticas Académicas de $3^{\circ}$ de la ESO, tomando como punto de partida la utilización del software Scratch para introducir de forma didáctica las funciones lineal, afín y cuadrática. La didáctica para su planteamiento se basa en una combinación de metodologías activas basadas en el método demostrativo, el aprendizaje cooperativo y la gamificación.

\section{Referencias}

Bocconi, S., Chioccariello A., Dettori G.; Ferrari A., \& Engelhardt K. (2016). Developing Computational Thinking in Compulsory Education, Implications for Policy and Practice. Publications Office of the European Union, Luxemburgo.

Corralero, N. (2011). Scratch. Programación fácil para educación primaria y secundaria, Revista Digital Sociedad de la Información, $n^{\circ}$ 29, 1-10.

Díez-Rioja, J., Bañeres-Besora, D. \& Serra-Vizern, M. (2017). Experiencia de gamificación en Secundaria en el Aprendizaje de Sistemas Digitales. Education in the Knowledge Society, 18(2), 85-105.

Ferrando, M. J. (2018). El Método Demostrativo, Universidad Internacional de Valencia, Valencia, España,

Forman G, \& Pufall P. B (1988). Constructivism in the Computer Age. Lawrence Erlbaum Associates, Estados Unidos. 
Johnson, D. W. y Johnson, R. T. (1995). Cooperative Learning. En J. H. Block, S. T. Everson y T. R. Guskey (Eds.), School Improvement Programs. A Handbook for Educational Leaders (pp. 25-56). Nueva York: Scholastic Inc.

Marji, M. (2014). Learn to Program with Scratch. San Francisco, California: No Starch Press. pp. xvii, 1-9, 13-15.

Quevedo-Gutiérrez, E., Vega-Moreno, D., Rodríguez-Cabrera, C., \& Quintana-Gil, E. (2016, febrero). Aprendizaje por proyectos aplicado a robótica submarina orientado a profesores y alumnos de secundaria. Comunicación presentada en Congreso Internacional de Tecnologías e Innovación Educativa, Universidad Internacional de Valencia, Valencia, España.

Papert S., \& Harel I. (1991). Constructionism. Ablex Publishing Corporation, Estados Unidos.

Salmerón, H., Gutiérrez-Braojos, C., Rodríguez, S., \& Salmerón, P. (2010). Influencia del aprendizaje cooperativo en el desarrollo de la competencia para aprender a aprender en la infancia. Revista Española de Orientación y Psicopedagogía, 21(2), 308-319.

Suárez, P., Andrés, C., \& Sarmiento-Porras, R. E. (2015). Estado del arte sobre experiencias de enseñanza de programación a niños y jóvenes para el mejoramiento de las competencias matemáticas en primaria. Revista mexicana de investigación educativa, 20(65), 607-641.

Wing J. (2010). Computational Thinking: What and Why?, The Link, The magazine of Carnegie Mellon University's School of Computer Science, 1-6.

Zamora, Á., \& Ardura, D. (2014). ¿En qué medida utilizan los estudiantes de Física de Bachillerato sus propios errores para aprender? Una experiencia de autorregulación en el aula de secundaria. Enseñanza de la Ciencias, 23(3), 253-268.

Zichermann, G., \& Cunningham, C. (2011). Gamification by Design: Implementing Game Mechanics in Web and Mobile Apps. Cambridge: O'Reilly Media. 